\title{
Auditory and visual confusions: Evidence against simple modality encoding hypotheses*
}

\author{
RAYMOND BRIGGS $\dagger$ \\ Oakland University, Rochester, Michigan 48063
}

\begin{abstract}
Using both recognition and recall responses, confusion and intrusion errors were obtained for briefly exposed 11-letter strings. The patterns of errors were sharply dependent upon experimental variables. In Experiment I Ss made auditory and visual intrusions with recall, but neither with recognition. In Experiment II increasing exposure time and eliminating a poststimulus cue primarily increased auditory confusions. This suggests that auditory and visual confusions reflect strategy-contingent recoding rather than modality-specific encoding.
\end{abstract}

Ss who are asked to report letters from a visual representation occasionally make "auditory confusions," i.e., they generate intrusions which sound like actually presented letters (Sperling, 1960; Conrad, 1964). Other evidence (Keele \& Chase, 1967; Laughery \& Harris, 1970; Kroll, Parks, Parkinson, Bieber, \& Johnson, 1970; Rollins \& Thibadeau, 1972) suggests the possibility of "visual confusions" as well.

The auditory confusions have led many (i.e., Sperling, 1960; Atkinson \& Shiffrin, 1968) to suppose that (1) visually presented letters must pass through a stage of auditory encoding and (2) letters must be transferred directly to this stage from a rapidly fading icon or be lost.

The visual confusions obtained in different experiments have often been interpreted as demonstrating the existence of a separate visual code lasting beyond the duration of iconic storage and separate from the auditory store (Murdock \& Walker, 1969; Henderson, 1972).

However, it is possible that such simple modality encoding hypotheses are premature. Existing experiments do not specify where between the presentation and response the confusion occurs. What is taken to be "encoding into memory" might be "retrieval for a response." Thus, a $\mathrm{S}$ might encode aurally and recode visually for a recognition response or encode aurally and make a recall response without recoding. In this case, an observed visual confusion would obscure the basic auditory encoding that was independent of the response. Alternatively, the $S$ might initially encode information abstractly, with no modality at all. As long as a modality-specific response was required, the subsequent auditory recoding for recall might lead one to assume that encoding was aural.

Such possibilities suggest a systematic search for the occurrence of both visual and auditory confusions in the

\footnotetext{
*This research was performed in partial fulfillment of the PhD degree at Cornell University, Ithaca, New York, under the supervision of Ulric Neisser.

tR equests for reprints should be sent to Raymond P. Briggs, Department of Psychology, Oakland University, Rochester. Michigan 48063.
}

same experiment. Since the occurrence of specific types of confusions might well depend on S's strategy, varying certain experimental conditions could change the relative frequency of visual and auditory confusions.

There were two methods of testing for auditory and visual confusions. In both, strings of letters greater than the span of apprehension (11 letters) were presented to Ss. In a recall version, Ss wrote down as many letters as they could in the correct serial position. Intrusion errors were scored. In the recognition version, a second string of letters was presented with one potential auditory or visual intrusion substituted.

Two experiments will be reported. In Experiment I auditory and visual confusions are compared between recognition (or one-item recall where visual coding had been demonstrated) and serial recall (where auditory coding had been demonstrated). In Experiment II an attempt was made to change the relative frequency of auditory and visual confusions in recognition by varying: (a) exposure duration, which might affect S's strategy by varying the time available for encoding, and (b) the presence or absence of a poststimulus cue, which might affect $S$ 's strategy for discovering a changed letter.

Under those conditions, simple modality hypotheses might predict: (1) auditory confusions would predominate in both recognition and recall. Visual confusions might occur in recognition (where visual coding is made more salient) but should be near chance in recall (where the response is closely linked to an auditory code). (2) Decreasing exposure duration should increase auditory confusions by making the coding more difficult, and the presence of a poststimulus cue might increase visual confusions if the $S$ could read from a rapidly fading icon.

An alternative approach would suggest that each new variant of the task changes the constraints on the $S$ and causes him to code differently. (1) When the $S$ must make a written recall response, he must involve both a serial coding response (auditory) and a recognition of his own writing (visual). This would suggest auditory and visual confusions. When the $S$ makes a judgmental (same-different) response in recognition, he need not 
Table 1

List of Embedded Targets Together With the Confusions Placed on the Answer Sheet

\begin{tabular}{cccccccc}
\hline \multicolumn{2}{c}{ Auditory } & & \multicolumn{2}{c}{ Visual } & & \multicolumn{2}{c}{ Neu:ral } \\
\cline { 7 - 8 } TAR & CON & & TAR & CON & & TAR & CON \\
\hline B & C & R & P & A & S \\
J & K & D & O & G & X \\
E & V & K & X & L & C \\
Z & D & W & M & M & Q \\
T & G & H & T & C & Y \\
X & F & O & Q & I & U \\
Q & U & P & R & U & Z \\
Y & I & F & E & V & O \\
\hline
\end{tabular}

Note $-T A R=$ target,$C O N=$ confusion

code information by modality at all. If the response is nearly immediate (with near perfect recoding), the $S$ could respond correctly without exhibiting auditory or visual coding.

If this is in fact happening, then auditory and/or visual confusions should appear if modality-specific recoding is made more salient. (2) Increasing exposure duration might encourage the $S$ to rehearse the information serially prior to the response; the absence of a poststimulus cue might encourage the $S$ to recode serially (to preserve order information) while searching for a changed letter. With a poststimulus cue, recoding is not as crucial, since $\mathbf{S}$ compares one cued target letter with the remembered letters.

\section{EXPERIMENT I}

\section{Method}

Subjects. Forty-seven college students run in small groups served as Ss.

Materials and Procedure. The apparatus consisted of a Kodak Carousel 500-W projector wired to a Hunter timer that controlled the exposure at $1.1 \mathrm{sec}$. The screen was a standard beaded screen (brightness at screen was $22 \mathrm{~mL}$ ). The lighting of the room was adjusted until the illumination of the projected string and the S's answer sheet seemed equivalent. The distance between $S$ and the screen varied from 92 to 132 in. (Ss who claimed lower visual acuity sat closer). The projected string of letters was $11 \frac{1 / 2}{2} 3 / 4$ in. high and varied between 7 and $11 \mathrm{deg}$ horizontally and 25 and 32 min vertically at the S's eye.

The experiment consisted of two phases: a recognition test (Part A) and a written recall test (Part B). Part A will be described first.

Materials. Two hundred and twenty pairs of letter strings (e.g., AGRSVQPYTLK and ZGRSNQPYTLK) were constructed. The first member of each pair, the "stimulus string," was photocopied for projection. The second member, or "test string," appeared on the S's answer sheet as described below. The strings were random groups of letters with the following constraints: (1) no repetitions of letters within strings, (2) no recognizable chunking of letters (i.e., SCH, OUL was not permitted; HCS, ULO was permitted), and (3) approximately equal occurrences of each letter of the alphabet at each serial position.

In one-third of the pairs of strings (72), the two members were identical ("same" pairs), while the other two-thirds ("different" pairs) differed by the substitution of a single letter in Serial Positions $1,3,5,7,9$, or 11 in the test string (see example above). The possible substitution in all trials was cued by means of underlining. The only obvious difference between the stimulus strings and the test strings was that one letter was underlined on the test string. The nature of the substitutions was carefully controlled. Of the 144 "different" trials, one-third (48) contained substitutions which looked but did not sound like the target letter $(\mathrm{F}: E)$; one-third contained substitutions which sounded but did not look like the target letter $(\mathrm{T}: G)$; and one-third contained substitutions which neither looked nor sounded like the target letter $(\mathrm{V}: \mathrm{O})$. All letters were used except "N" (see Table 1). Letters were considered aurally similar if their letter name had a phoneme in common with another letter name, a procedure justified by the work of Wickelgren (1965). Letters were considered visually similar when their latencies were longer than a criterion $(350 \mathrm{msec})$ on a same-different reaction time test. In the experiment which generated these latencies, Gibson, Yonas, and Shapiro ${ }^{1}$ showed that visual confusions and reaction time were positively correlated. The neutral controls were selected to be as different as possible both in name and in form.

The sequence of 21611 -letter pairs was ordered randomly with the following constraints: (1) Targets could not occur in the same serial position more than twice in succession, and (2) strings with the same substitution could occur no more than twice in succession.

Answer Sheet. The answer sheet was composed of 11 pages of 11-letter strings, with 20 strings per page. These test strings always had an underlined letter. Below each string on the answer sheet were the words "same" and "different" (see Fig. 1).

Each string on the answer sheet was covered individually by a black tab of paper when the experiment began. Half of the tabs were pulled off from the left and half were pulled off from the right.

Procedure. Ss were run in groups of 12 or fewer. They were read a prepared set of instructions which explained what a trial consisted of and were given an opportunity to ask questions. In addition, they were given four practice trials. Finally, they were asked to indicate uncertainty by circling uncertain trials on their answer sheets. They were instructed: "Try to indicate uncertainty. It is hoped that you can distinguish between your guesses based upon information and your guesses based upon nothing."

A trial consisted of the following: The E said, "ready." There was a short pause. The stimulus string was exposed by projector for $1.1 \mathrm{sec}$. The $\mathrm{S}$ removed a black tab, exposing the test string. The S checked "same" or "different" and either did or did not circle the word to indicate uncertainty.

The intertrial interval was determined by the group. A regular rhy thm developed, and the average trial lasted about 9 sec. The entire experiment lasted about $55 \mathrm{~min}$.

Part B Experiment. The experiment for Part B was identical to that for Part A, except that there was no target letter embedded, and the answer sheet consisted of blanks to be filled in by the $S$ using written recall. The answer sheet looked like this: 1. "In the next part, you will write in as many of the letters as you can remember. Put the correct letter in the correct blank." Part B always followed Part A.

\section{Results}

We will first consider the data from Part $B$, in which the $S$ used written recall.

Accuracy. Accuracy was scored by correct letter in the string, with 5.35 letters per string. An intrusion error

$$
\begin{array}{lllllllllll}
Z & G & \mathbf{R} & \mathbf{S} & \underline{N} & \mathbf{Q} & \mathbf{P} & \mathrm{Y} & \mathbf{T} & \mathbf{L} & \mathbf{K}
\end{array}
$$

Same Different

Fig. 1. Typical entry for answer sheet. 
Table 2

Confusion Matrix: Experiment II. Full Set of Observed Intrusions*

\begin{tabular}{|c|c|c|c|c|c|c|c|c|c|c|c|c|c|c|c|c|c|c|c|c|c|c|c|c|c|c|c|}
\hline \multirow{2}{*}{$\begin{array}{c}\text { Letter } \\
\text { Presented }\end{array}$} & \multicolumn{26}{|c|}{ Confusions Made by Ss } & \multirow[b]{2}{*}{ Total } \\
\hline & A & $\mathbf{B}$ & $\mathrm{C}$ & $\mathrm{D}$ & E & $\mathrm{F}$ & $\mathbf{G}$ & $\mathbf{H}$ & 1 & $J$ & $\mathbf{K}$ & $\mathbf{L}$ & $\mathbf{M}$ & $\mathbf{N}$ & $\mathrm{O}$ & $\mathbf{P}$ & $Q$ & $\mathbf{R}$ & $S$ & $\mathrm{~T}$ & $\mathrm{U}$ & $\mathrm{V}$ & W & $\mathrm{X}$ & $\mathrm{Y}$ & $\mathrm{Z}$ & \\
\hline $\mathbf{A}$ & & & & 1 & 1 & & & & & 1 & & & & & & & & & & & & & & & 1 & & 4 \\
\hline B & 1 & & & & & & & & & 1 & & 6 & 3 & 1 & & 2 & & 1 & 1 & 2 & & & 1 & & & & 19 \\
\hline $\mathrm{C}$ & & & & & 2 & 1 & 2 & & 1 & & 1 & & & 3 & & 1 & & & 1 & & & & & & & 2 & 14 \\
\hline D & & 1 & & & & 1 & & 1 & 1 & & & 1 & & 1 & 2 & & & 2 & & 4 & & 1 & & & & & 15 \\
\hline $\mathbf{E}$ & & & & & & 1 & 1 & & 1 & 1 & & & & & & 1 & & & & 1 & & & & & & 1 & 7 \\
\hline $\mathbf{F}$ & 2 & 1 & 1 & & 1 & & 2 & & 1 & & 1 & & 2 & & & & 1 & & 1 & & 1 & 1 & & 5 & & 1 & 21 \\
\hline $\mathrm{G}$ & & 2 & 1 & 1 & & & & 2 & & & & & & & 2 & 1 & 1 & 2 & & & & & & 1 & & & 13 \\
\hline $\mathrm{H}$ & 1 & & & & & & & & 1 & & & 2 & 1 & 2 & 1 & 2 & & 2 & & 1 & & & & 3 & 1 & & 18 \\
\hline I & 1 & & & & & & & & & & & & & & & & & & & 2 & & & & & & & 3 \\
\hline $\mathbf{J}$ & 2 & & & 1 & & & 2 & & 2 & & 1 & 4 & 2 & 1 & 3 & & & 3 & & 3 & & & & & 1 & & 23 \\
\hline $\mathbf{K}$ & 1 & & & & & & 1 & & 1 & & & & 1 & & 1 & 2 & 1 & & 1 & & & & & 1 & 1 & 1 & 13 \\
\hline L & & & 1 & & 2 & 2 & & 2 & 1 & & 3 & & & 1 & & & 3 & 1 & 1 & & & & & 2 & & 1 & 20 \\
\hline $\mathbf{M}$ & 1 & & & 1 & 1 & & & 2 & & & & & & 1 & & 1 & & 1 & 1 & & 1 & & 2 & & 1 & 1 & 14 \\
\hline $\mathbf{N}$ & 1 & 1 & & & 1 & 1 & & 1 & & & 1 & & 1 & & & & & & 2 & & & 2 & & & & & 10 \\
\hline 0 & & 1 & & & & & & & 1 & 1 & & 1 & & & & & 1 & & & & 1 & & & & & & 6 \\
\hline $\mathbf{P}$ & & & & & & & & & & & & & & & 1 & & & & 1 & & 1 & & & & 1 & 1 & 5 \\
\hline$Q$ & & & & 1 & & & 1 & & & 1 & & & 3 & & & 1 & & 1 & & & 1 & & & & & & 9 \\
\hline $\mathbf{R}$ & 1 & 1 & & & 1 & & & & 1 & & & & 1 & 1 & 1 & & & & & & & & & & 2 & & 9 \\
\hline$S$ & & & & & & 1 & & & & 2 & 1 & 1 & & & & 1 & & & & & 2 & & & & & 1 & 10 \\
\hline $\mathrm{T}$ & 1 & & & & & & 1 & & & 1 & & 1 & 1 & & & 2 & 1 & 2 & & & & & & & 1 & & 11 \\
\hline $\mathbf{U}$ & & & & & & & & & & & & & & & & & 1 & 1 & & & & & & & & & 2 \\
\hline V & & & & & & & & & & & & & 1 & & & & & & & 1 & 3 & & & & & 1 & 6 \\
\hline W & & & & & & & 1 & & & & & & 1 & & & & 1 & & 1 & 1 & & & & & & & 5 \\
\hline $\mathrm{X}$ & & & & & & & & & & & & 1 & & & & 1 & 1 & 1 & & & & & & & & & 4 \\
\hline $\mathrm{Y}$ & 2 & 1 & & & & & & & & 2 & & 1 & & 2 & 1 & & & & 1 & & & & 1 & & 1 & & 12 \\
\hline $\mathrm{Z}$ & & & 1 & 2 & & & & & & & & & & 1 & 2 & & & & 4 & & 2 & & & 2 & & & 14 \\
\hline
\end{tabular}

*Blank spaces mean no confusions.

was defined as any reproduced letter that was not a member of the presented string. Intrusions were scored as substitutions for letters in the stimulus string at whatever serial position they appeared in the answer sheet. The Ss made an average of 5.9 intrusions in 53.5 responses, or 1 intrusion per 9.06 responses. These data were organized into the confusion matrix of Table 2.

To test whether or not these intrusions could have occurred by chance alone, all possible pairs of capital letters were partitioned on the basis of auditory and visual similarity into four groups: (1) aurally similar pairs $(\mathrm{N}=86)$, (2) visually similar pairs $(\mathrm{N}=86)$, (3) aurally and visually similar pairs $(\mathrm{N}=30)$, and (4) aurally and visually distinct pairs $(\mathrm{N}=448)$.

Since Groups 1, 2, and 3 contain pairs which are similar and Group 4 contains pairs which are distinct, it is possible to compare the number of confusions made for similar and distinct pairs. This creates three ratios: (a) visually similar/distinct $(86 / 448)=0.192$, (b) aurally similar/distinct $(86 / 448)=0.192$, and $(c)$ aurally and visually similar/distinct $(30 / 448)=0.067$. Each of these

Table 3

Total Number of Errors, Total Number of Certain Errors, Total Percent Errors, Certain Percent Errors

\begin{tabular}{lccc}
\hline & Auditory & Visual & Neutral \\
\hline Total Number of Errors & 569 & 539 & 552 \\
Total Percent Errors & 25.2 & 23.9 & 24.5 \\
Total Number of Errors & 367 & 324 & 343 \\
on "Certain" Trials & 19.4 & 18.0 & 19.4 \\
\hline
\end{tabular}

ratios reflects the proportion of expected confusions if confusions called "similar" and confusions called "distinct" are equally likely. Any observed proportion greater than $a, b$, or $c$ would reflect the greater confusability of "similar" letters. To test this, two ratios are constructed for each of the 26 letters in the alphabet. One ratio $(\mathrm{X})$ reflects the expected proportion based on the partitioning of the 650 cells in the matrix. The other $(Y)$ reflects the actual intrusions in the matrix. If the first proportion (X) is greater than the second $(Y)$, then a "-." is indicated. If the second $(Y)$ is greater, a " + " is indicated.

If similarity makes no difference, there should be as many pluses as minuses. If similarity matters, there should be significantly more pluses. The test for visual similarity showed $19+$ and $7-$. This difference is significant $(\mathrm{p}<.05$, one-tailed by a sign test). The test for aural similarity showed $16+$ and $5-$, also a significant difference $(p<.05$, one-tailed by a sign test). The auditory-visual similarity test $(9+$ and $8-)$ failed to reach significance.

The data from Part $\mathbf{A}$ involving recognition responses will now be considered.

It had been expected that $S$ would make more auditory confusions than control confusions and more visual confusions than control confusions. As Table 3 shows, this did not happen. The number of errors made was virtually identical for auditory, visual, and neutral substitutions. When all circled trials (uncertain responses) were eliminated, the result was unchanged. 


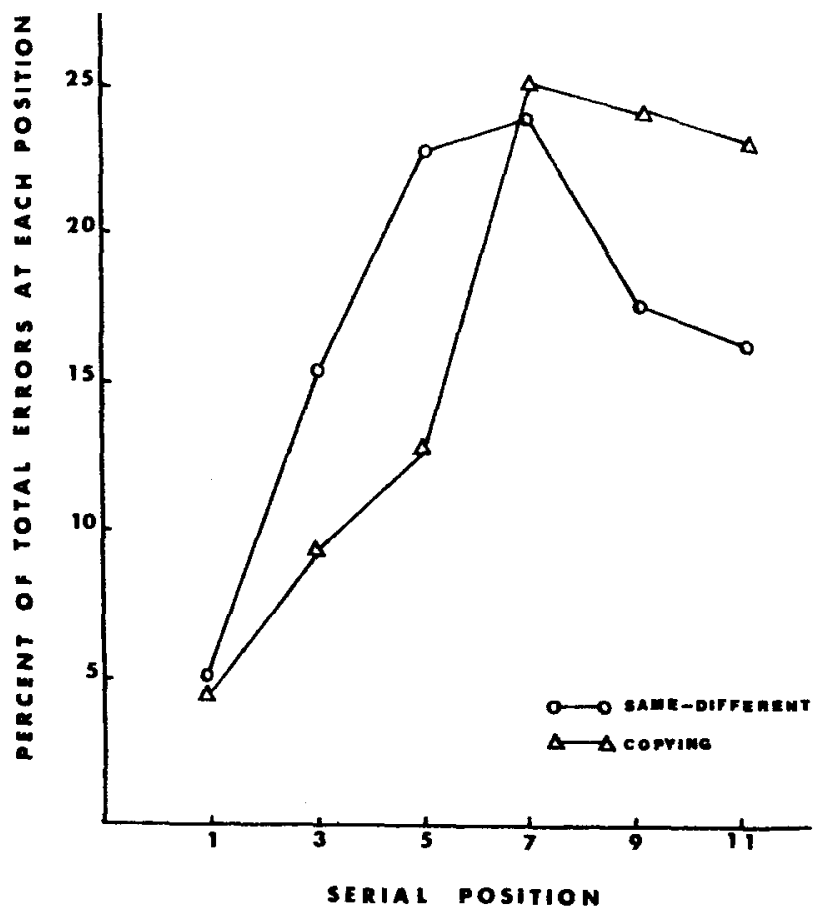

Fig. 2. Errors by serial position for recognition and recall.

Finally, sign tests across Ss (analogous to those performed on the matrix in Part B) comparing the number of auditory or visual confusions with the number of neutral control confusions did not even approach significance.

Even assuming a discrimination hypothesis, one might expect some visual confusions. That is, Ss might make more confusions between visually similar and dissimilar letters, not because they made errors in memory but because they originally saw the letter incorrectly. ${ }^{2}$ The data indicate slightly more neutral control confusions than visual confusions, the opposite of what might be expected.

Finally, Parts A and B will be compared. Figure 2 is a plot of the serial position data from Part A (recognition) and Part B (recall). Although the Part B curve is skewed to the right with respect to the Part $A$ curve, the difference between the shape of the two curves does not even approach significance with a Kolmogorov-Smirnoff two-sample test. More important, the Ss' accuracy on the recognition and recall tasks is significantly correlated ( $p<.05$, two-tailed $t$ test), with a correlation coefficient of .29 .

\section{Discussion}

The simplest explanation of the data in this experiment hinges on the assumption that Ss are doing very similar things in recognition and recall. We know that the Ss' recall in this experiment is 5.35 letters. It would be easy to assume that the $S$ also encodes $5+$ letters in the recognition task. When any of the 5+ encoded letters appear as targets (half of the time), the $S$ gets them correct. On trials where more encoded letters appear as targets, the $\mathrm{S}$ guesses randomly. This would generate a recognition accuracy of $75 \%$, slightly less than the actual $76.2 \%$ accuracy of Ss in Part A.

In the present experiment, the Ss were not forced to rely upon either auditory or visual information. They seem to have used both for recall (although the effect was small) and neither for recognition.

It is the lack of confusions in recognition which is most difficult for any simple modality encoding hypothesis to explain. Ss either were encoding abstractly (or amodally) or were unaffected by the distracting confusions substituted by the $\mathrm{E}$.

\section{EXPERIMENT II}

Experiment I suggests that Ss were coding by modality with recall but not recognition. It might be, however, that Ss were merely unaffected by the auditory and visual substitutions used.

If $\mathrm{Ss}$ were encoding abstractly and recoding for immediate recognition, it might be possible to induce modality-specific recoding. Increasing exposure would give the $\mathrm{S}$ time to organize information prior to the response. If this organization took the form of auditory rehearsal or visual recoding, then modality-specific confusions should occur.

Another possible way to induce at least auditory coding would be to eliminate the poststimulus cue used in the recognition experiment (Experiment I, Part A). This would lead the $S$ to compare more letters, a procedure consistent with serial aural coding.

\section{Method}

Subjects. Ss were college students run individually. There were two groups, with 12 Ss per group.

Apparatus and Conditions. Apparatus and conditions were similar to those of Experiment I, except that Ss were run individually $92 \mathrm{in}$. from the screen. The $\mathrm{S}$ was seated in front of a button with a box marked "same" on his left and a box marked "different" on his right.

Materials. Three identical (for durations of $0.5,1.0$, and $2.0 \mathrm{sec}$ ) sets of cards were constructed for the "cued condition," and three other sets (identical to each other) were constructed for the "not cued condition." As before, all were constructed in pairs, were 11 letters long, and contained auditory, visual, and neutral substitutions. There were 144 cards in each set. For 72 cards in each set, the stimulus and test cards were identical. Of the 72 that were different, 36 contained neutral control substitutions, 18 contained visual substitutions, and 18 contained auditory substitutions. The complete list of targets and substitutions is displayed in Table 4.

Strings were typed on the cards and arranged into a pile containing cards alternating with blanks. "Same" and "different" were not written below each string; instead, Ss placed each card in a box marked either "same" or "different."

The only difference between the materials for the cued and not cued condition was that a critical letter was underlined on each card in the cued condition (as in Experiment I), whereas nothing was underlined in the not cued condition.

Procedure. Both the materials and the exposure times were organized into blocks. Auditory substitutions were paired with matched neutral substitutions and these "different" trials were 
Table 4

Auditory and Visual Substitutions with Matched Controls by Blocks

\begin{tabular}{cccccc}
\hline Target & $\begin{array}{c}\text { Substitu- } \\
\text { tion }\end{array}$ & \multicolumn{3}{c}{$\begin{array}{c}\text { Substi- } \\
\text { Target } \\
\text { tution }\end{array}$} \\
\hline & & \multicolumn{2}{c}{ Cued Condition } & & \\
K & & X & A & B \\
E & Visual & F & H & Neutral & L \\
V & & N & I & & Z \\
J & & K & L & A \\
T & Auditory & G & O & Neutral & Y \\
Z & & D & Z & & M \\
& & Not-Cued Condition & & \\
R & & B & S & & W \\
N & Visual & V & U & Neutral & H \\
M & & W & Y & O \\
X & & F & B & I \\
C & Auditory & D & G & Neutral & U \\
P & & C & J & R \\
\hline
\end{tabular}

matched with an equal number of "same" trials. An identical procedure was followed for visual substitutions. Thus, in each block there were 18 trials containing experimental substitutions, 18 trials containing neu tral controls, and 36 trials in which no substitu tions were made.

Each $S$ was presented the same auditory and visual block three times, at $0.5,1.0$, and $2.0 \mathrm{sec}$. The three time blocks and the order of the auditory and visual blocks were counterbalanced across Ss. One group of Ss was run with cued test cards, as in Experiment I; the other group was run without any underlined letter on the test card

Ss were run as before, except that they were given rest pauses to keep the blocks separate. After each exposure duration interval, Ss left the room while the next set of cards and duration intervals were prepared. Ss were not asked to indicate uncertainty, and there were no practice trials.

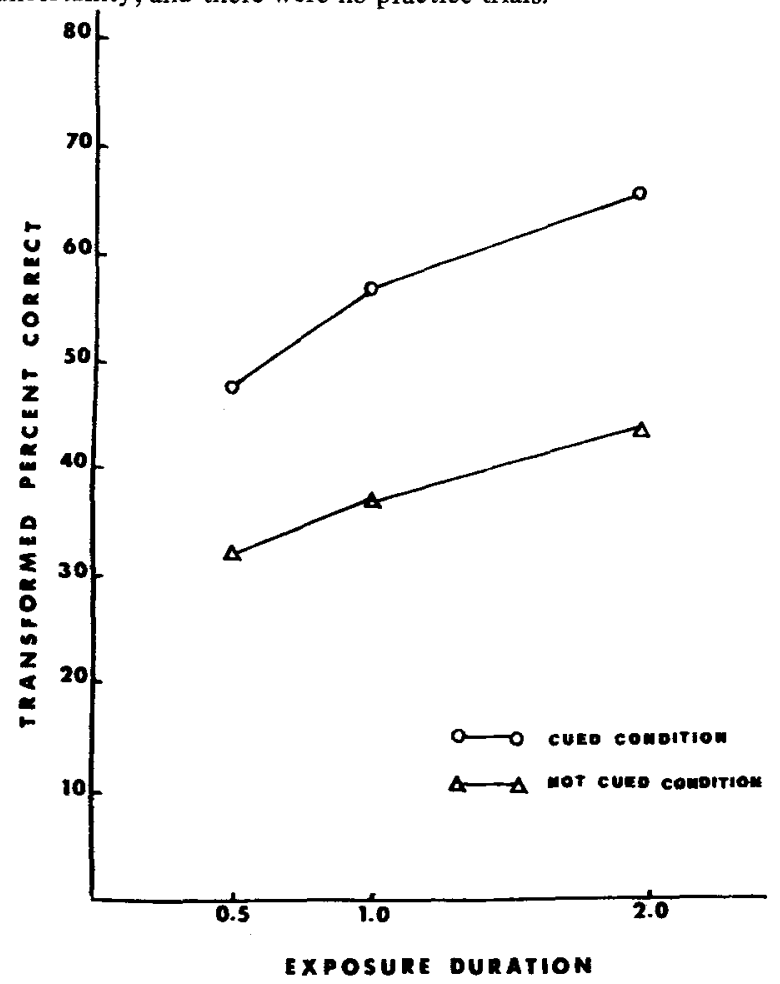

Fig. 3. Accuracy of $\mathrm{Ss}$ in the cued and not cued conditions at three exposure durations.

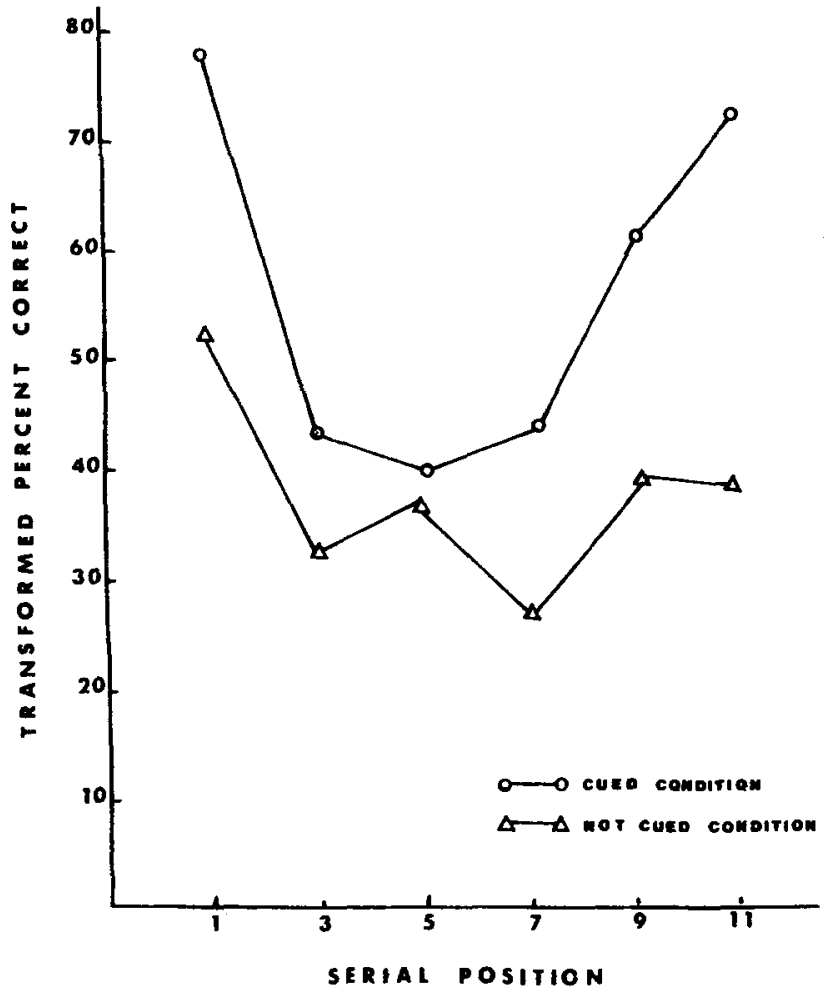

Fig. 5. Auditory and visual proportions for both cued and not cued conditions.

Trials were initiated by the $\mathrm{S}$. He pressed the button in front of him, which activated the projector. Following exposure of the projected string, the Ss turned over a blank card, exposing the test string. The test string was placed into a box marked either "same" or "different."

\section{Results}

Accuracy. Recognition data for both the cued and not cued conditions were transformed algebraically to adjust for chance. Figure 3 shows a large difference in accuracy between the cued and not cued condition, as well as an increase in accuracy due to exposure duration. Both the effects of exposure duration $(F=30.9, p<.001)$ and cueing $(F=71.8, p<.0001)$ are easily significant by an analysis of variance. It is interesting that the poststimulus cue aids accuracy more than a fourfold increase in exposure time.

Figure 4 shows the serial distribution of the cued vs not cued accuracy data. The main difference between the two conditions seems to appear at the ends, which exhibit a clear serial position effect only with the cued trials.

Confusions. The main question for this experiment was whether or not Ss could be affected by auditory or visual substitutions. Trials on which the $S$ incorrectly responded "same" were categorized as representing a "visual confusion" (V), an "auditory confusion" (A), or a neutral control confusion (C). Since the experimental design provided equally as many opportunities for each type of confusion, the tendency to make auditory and visual confusions could be indexed by $A /(A+C)$ and 


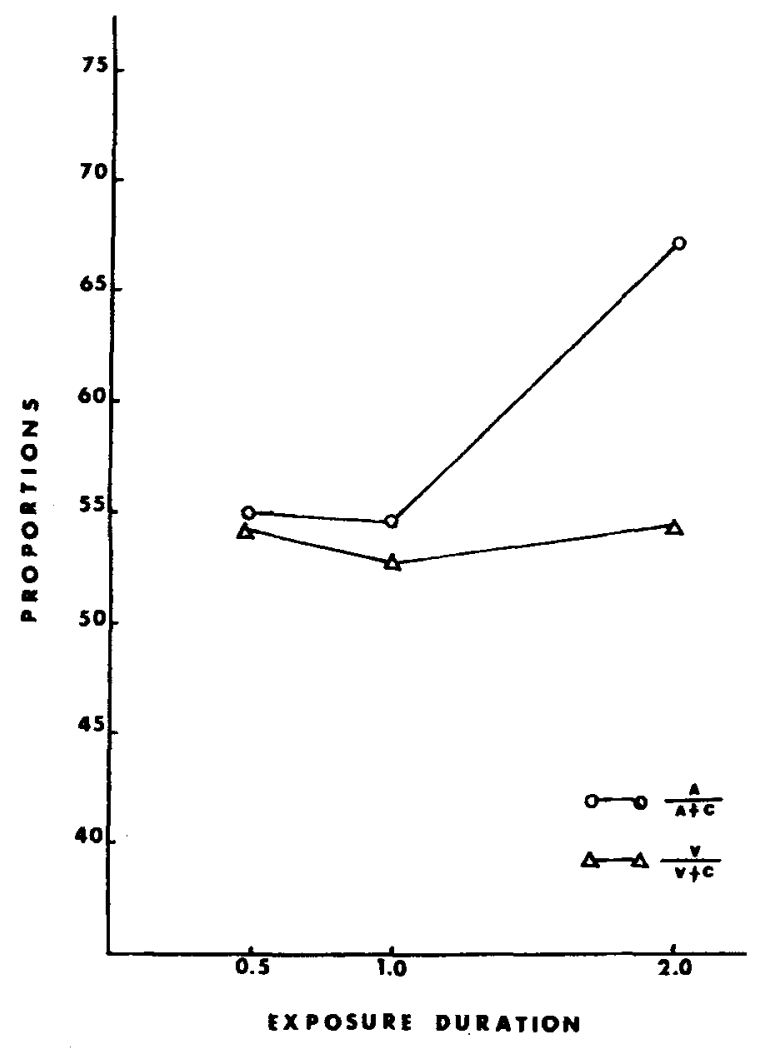

Fig. 4. Accuracy by serial position: Cued vs not cued data.

$\mathrm{V} /(\mathrm{V}+\mathrm{C})$, respectively, for each $\mathrm{S}$.

Figure 5 shows the overall pattern of results, combining both the cued and noncued data. Auditory and visual proportions do not differ from chance or each other at $0.5 \mathrm{sec}$ or $1.0 \mathrm{sec}$ (thus replicating Experiment I).

At $2.0 \mathrm{sec}$ Ss almost inevitably make auditory confusions (three confusions of four are auditory in the auditory blocks). The effect shows up in almost every S's data (22 of 24) and is significantly greater than the also significant visual proportion according to a $t$ test $(p<.05)$. Although the visual proportion does not change in magnitude appreciably with exposure duration, it stabilizes and reaches significance across Ss by a sign test $(p<.05)$ at $2.0 \mathrm{sec}$.

The pattern of results is slightly different for the cued and not cued conditions. The cued condition shows larger visual effects and a larger shift at $2.0 \mathrm{sec}$ in the auditory condition. The not cued condition consistently showed more auditory than visual confusions at all exposure durations, although the effect was small.

One other difference between the cued and not cued conditions was that Ss had different response biases. Cued Ss made $61 \%$ of their erroneous responses on trials in which the stimulus and target were identical. Not cued Ss made $63 \%$ of their erroneous responses on trials in which the stimulus and target were different.

\section{DISCUSSION}

It is clear that both manipulations made a difference.
Increased exposure time produced both better accuracy and significant proportions of auditory and visual confusions. Cued Ss performed better, shifted auditory and visual proportions more, and exhibited a big serial position effect.

\section{IMPLICATIONS AND CONCLUSIONS}

The pattern of results in Experiments I and II is very difficult to reconcile with simple modality encoding hypotheses. At the same exposure with similar materials, Ss indicated both auditory and visual confusions with recall and neither with recognition. The presence of auditory and visual confusions in recognition can be obtained by simply doubling the exposure time to $2.0 \mathrm{sec}$. The relative proportion of auditory and visual confusions can be altered by simply eliminating the cue for the target string.

There is no clear evidence in either of the experiments that any of the auditory or visual confusions reflect encoding. The absence of confusions at short exposure durations and the large increase in auditory confusions with increases in exposure duration suggest that modality-specific coding requires time. Thus, the best interpretation of the confusions generated in these experiments is that they reflect recoding rather than encoding. Until some procedure can distinguish more precisely between encoding and retrieval processes with a variety of tasks, it is perhaps best to withhold judgment as to the exact nature of the encoding of information. The possibilities seem to grow.

\section{REFERENCES}

Atkinson, R. C., \& Schiffrin, R. M. Human memory: A proposed system and its control processes. In $K$. W. Spence and J. T. Spence (Eds.), The psychology of learning and motivation. Vol. 2. New York: Academic Press, 89-195.

Conrad, $R$. Acoustic confusions in immediate memory. British Journal of Psychology, 1964, 55, 75-83.

Henderson, L. Visual and verbal codes: Spatial information survives the icon. Quarterly Journal of Experimental Psychology, 1972, 24, 439-447.

Keele, S. W., \& Chase, W. G. Short-term visual storage. Perception \& Psychophysics, 1967, 2, 383-386.

Kroll, N. E., Parks, J., Parkinson, S. R., Bieber, S. L., \& Johnson, A. L. STM while shadowing: Recall of visually and aurally presented letters. Journal of Experimental Psychology, 1970, 85, 220-224.

Laughery, K. R., \& Harris, G. J. Visual and auditory intrusion errors in short-term memory. Journal of Experimental Psychology, 1970, 85, 101-106.

Murdock, B., \& Walker, K. D. Modality effects in free recall. Journal of Verbal Learning \& Verbal Behavior, 1969, 8, 665-676.

Rollins, H. A., \& Thibadeau, R. The effects of auditory shadowing on recognition of information received visually. Memory \& Cognition, 1973, 1, 164-168.

Sperling, $G$. The information available in brief visual presentations. Psychological Monographs, 1960, 74(11).

Wickelgren, W. A. Acoustic similarity and intrusion errors in short-term memory. Journal of Experimental Psychology, $1965,70,102-108$.

\section{NOTES}

1. Gibson, E. J., Yonas, A., and Shapiro, F. Confusion matrices for graphic patterns obtained with a latency measure. Unpublished paper, Cornell University, 1968.

2. I am indebted to Julian Hochberg for explicating this possible interpretation of visual confusions.

(Received for publication December 20, 1973; revision received $F$ ebruary 7,1974 .) 